\title{
Tear trough - Anatomy and treatment by autologous fat grafting
}

\section{Chang Yung Chia}

Reconstructive Microsurgery Department of the Hospital dos Servidores do Estado do Rio de Janeiro, Rio de Janeiro, Brazil

\begin{abstract}
Tear trough is the main irregularity at midface, of which treatment is difficult. There is no agreement in literature about its anatomy and best treatment. The author presented an anatomical study and personal autologous fat grafting technique for tear trough treatment. Anatomical dissections were done on two fresh cadavers to examine the skin, subcutaneous, muscle and bone layers, spaces, and attachments. Safety and efficacy were evaluated via retrospective analysis of the last 200 consecutive procedures performed by the author. Tear trough is caused by the abrupt transition of the palpebral orbicular oculi muscle (OOM) (i.e., thin skin without subcutaneous fat compartment) to the orbital OOM (i.e., thicker skin with malar fat compartment). The tear trough region is located at the OOM bony origin at the medial canthus where no specific ligament was found. The grafted fat volume stabilized at two or three months after the procedure, instead of six months as stated in literature, with excellent results and no severe complications. Tear trough is a personal characteristic, a natural anatomical depression caused by subcutaneous irregularity and can worsen with age. The lack of volume is not effectively corrected by surgeries and thus it must be filled. Fat grafting has several advantages over alloplastic fillers, although it may be more difficult. Fat graft is autologous and abundant, and tissue transplantation could enhance skin quality. Fat grafting is a simple, safe, and effective solution for adding extra volume to correct the deflation phenomenon of the midface aging process. There is no specific anatomical plane for volume injection; the fat graft must be evenly distributed in the deep and superficial plane for uniformity.
\end{abstract}

Keywords: Tear trough; fat graft; lid cheek junction; facial anatomy; aging face

Citation: Chang YC. Tear trough - Anatomy and treatment by autologous fat grafting. J Surg Dermatol 2016; 1(2): 116-122; http://dx.doi.org/10.18282/jsd.v1.i2.45.

Correspondence to: Chang Yung Chia, Reconstructive Microsurgery Department of the Hospital dos Servidores do Estado do Rio de Janeiro, Rio de Janeiro, RJ, Brazil, changplastica@gmail.com.

Received: $16^{\text {th }}$ February 2016; Accepted: $5^{\text {th }}$ April 2016; Published Online: $14^{\text {th }}$ July 2016

\section{Introduction}

The most important characteristic in the concept of beauty and youth in facial contouring is the uniformity and regularity of the malar prominence that is in smooth continuity with the orbitopalpebral region (Figure 1). The main irregularities of this region are the lateral lid-cheek junction, the central "V" deformity, and especially the marked medial tear trough ${ }^{[1]}$. Inferior palpebral fat compartments may be also prominent enhancing the upper volume and causing a deeper impression of the periorbital sulcus. The inferior palpebral skin is usually darker, further highlighting the deformity (Figure 2).

These are definitely personal characteristics since childhood (Figure 3) and could worsen with age ${ }^{[2]}$, but these are not "deformities". It could also be iatrogenic through excessive release of the inferior palpebral fat pad during blepharoplasties (Figure 4).

It has been known that the aging process is not a gravitational descent of facial or body tissue. Aging is genetically programmed, with quality and quantity alterations of tissues. In the face, the skin loses collagen and elastic fibers, decreasing in vascularity and appendages,

Copyright (C) 2016 Chang YC. This is an Open Access article distributed under the terms of the Creative Commons Attribution-Non Commercial 4.0 International License (http://creativecommons.org/licenses/by-nc/4.0/), permitting all non-commercial use, distribution, and reproduction in any medium, provided the original work is properly cited. 


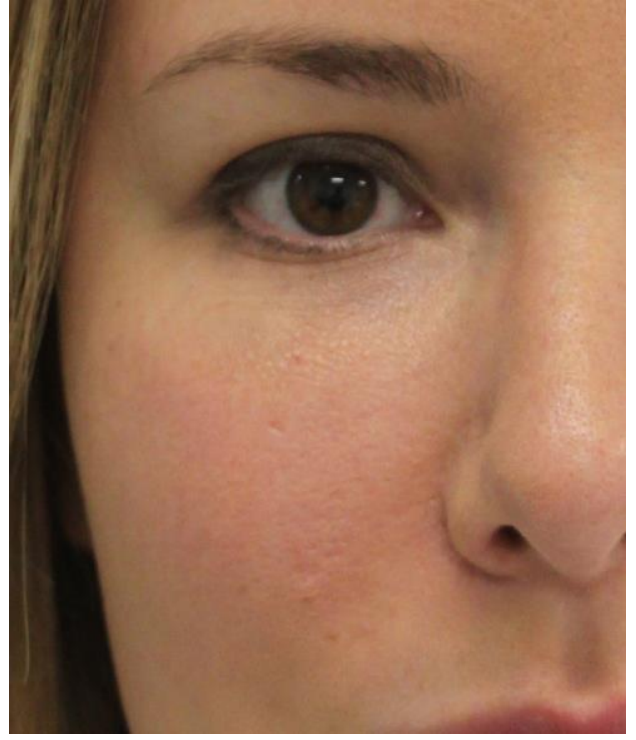

Figure 1. The beauty pattern: Regular malar prominence, in continuity with the orbitopalpebral region, forming one aesthetic unit

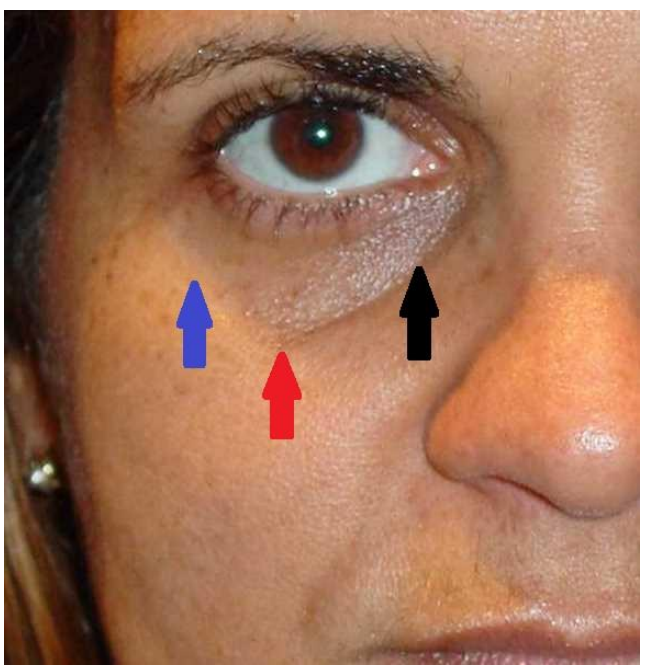

Figure 2. Periorbital deformities: Tear trough (black arrow), central "V" deformity (red arrow), and palpebromalar sulcus (blue arrow)
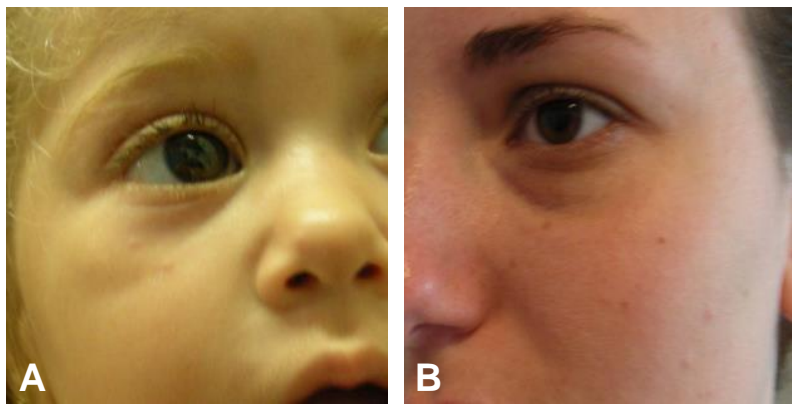

Figure 3. A three-year-old child (A) and a 23-year-old girl with tear trough (B)
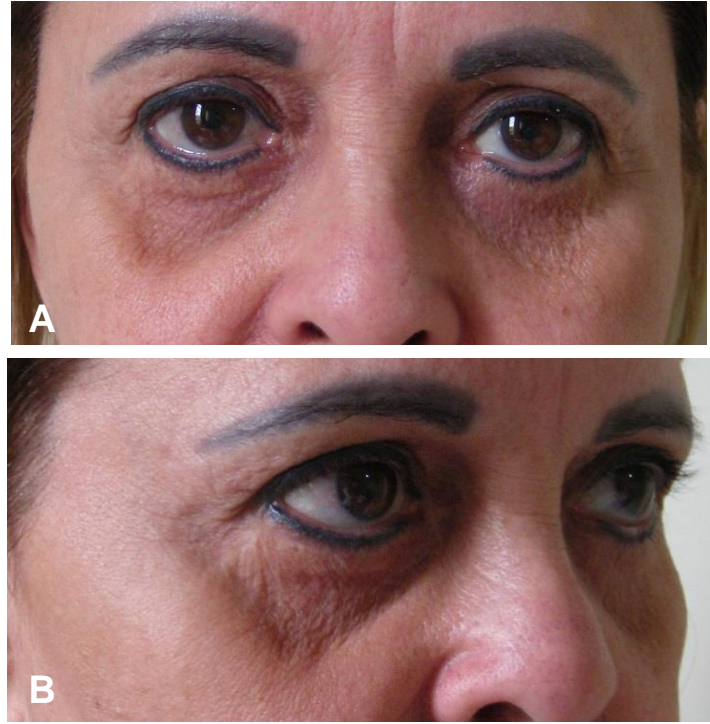

Figure 4. Excessive release of the inferior palpebral fat pads during previous blepharoplasties, front view (A), oblique view (B)

thus becoming thinner and flaccid. Fat compartments change the volumetric distribution, and the facial bones are in continuous remodeling throughout life ${ }^{[3-10]}$. Hence, in the midface, there is a deflation phenomenon. Treatment must be directed against these targets ${ }^{[11-15]}$.

However, there are disagreements in literature regarding the anatomy of these landmarks. The present work presented an anatomical study via cadaveric dissection and personal autologous fat graft technique for tear trough treatment.

\section{Methods}

\section{Anatomical study}

The study was made via analysis of surgical dissection of two fresh male cadavers, both estimated to be about 40 years old. Dissection was performed layer by layer, with a particular interest on the skin thickness, the presence of subcutaneous fat, the position of muscles, and bone attachments.

\section{Fat grafting technique}

Patient evaluation and surgical plans were made in the orthostatic position. Facial deformities were marked. The inferior palpebral fat pads can be highlighted but rarely have indication for release nowadays and hence must be carefully valued. The ideal malar projection should be slightly more prominent than the palpebral region, with a smooth curve (Figure 5A). When the fat pad is more prominent than the eye, it must be released and the de- 
pression around the eyes be filled (Figure 5B). When the fat pad is evidenced by the depression around the eyes but is not projected, all tissues must be filled without surgical release of the fat pad (Figure 5C).
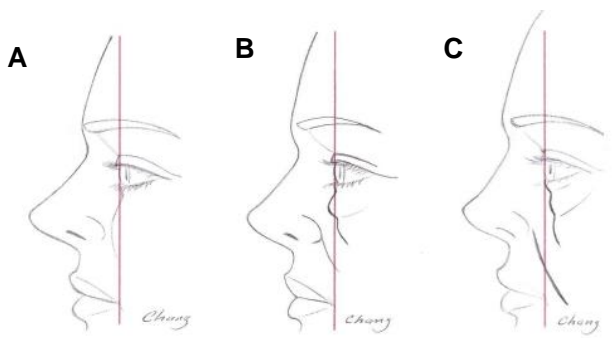

Figure 5. The ideal profile (A); Inferior palpebral fat pad more prominent than the eye: An indication for surgical release and filling around the depression (B); Fat pad highlighted by tissue depression around the eyes: No indication for release, only filling $(\mathrm{C})$

Periorbital filling is the first procedure done in a facelift surgery, before the appearance of edema. The choice of region of donor fat can be according to the patient's or physician's preference. If general anesthesia is used, no infiltration is made. If anesthesia is local, the solution to be used would be lidocaine $0.4 \%$ with adrenaline 1:200,000, injected evenly in the subcutaneous at a proportion of $1: 1$. On the face, the infraorbital and zygomatic facial nerves are blocked with a minimum quantity of anesthetic solution (Figure 6).
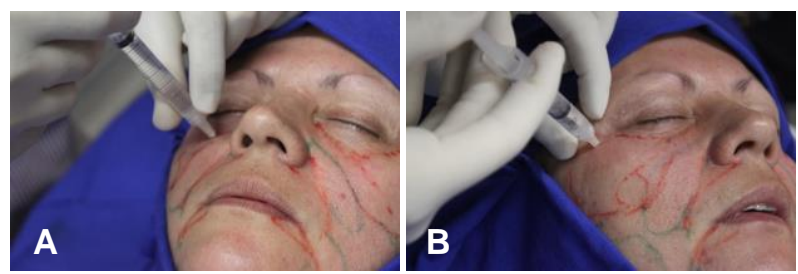

Figure 6. Infraorbital nerve block (A); Zygomatic facial nerve block (B)

Fat was harvested via negative pressure, either pumpassisted or by syringes, using a 2- or 3-mm diameter conventional cannula. Harvested fat was decanted without washing until three segments are defined. Supernatant oil and its underlying liquid were separated, and the fat was passed to a 1-mL syringe (Figure 7). The fat grafting technique used is called slow retro-injection, with small quantities or "fine tunnels".

A cannula of $1.2 \mathrm{~mm}$ in diameter with one lateral hole at the tip can be used. For the periorbital region, the entrance point is several millimeters under the orbital rim, below the lowest point of the "V" deformity. This point allows medial access for tear trough correction, lateral and central access for lid check junction and "V" deformity, and inferior access for malar augmentation. The fat graft was retro-injected first at the supraperiosteal plane (Figure 8) and subsequently at more superficial planes, always in small quantities with each passage, until the desired volume and superficial regularity was reached (Figure 9).
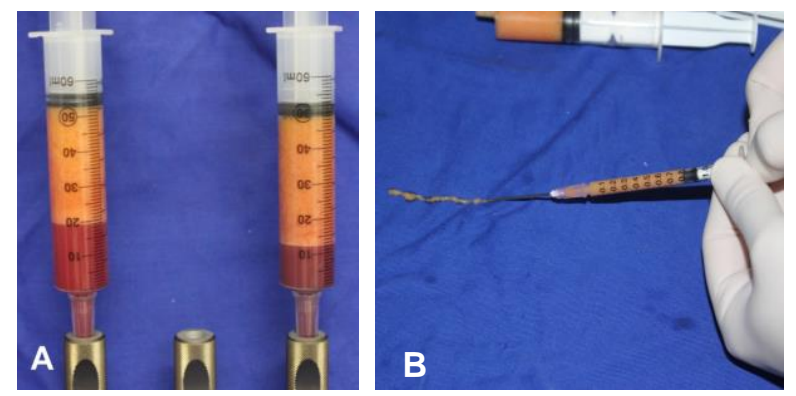

Figure 7. Decanting the suctioned fat until three segments are defined (A); Retro-injection technique with $1 \mathrm{~mL}$ syringe and microcannula (B)

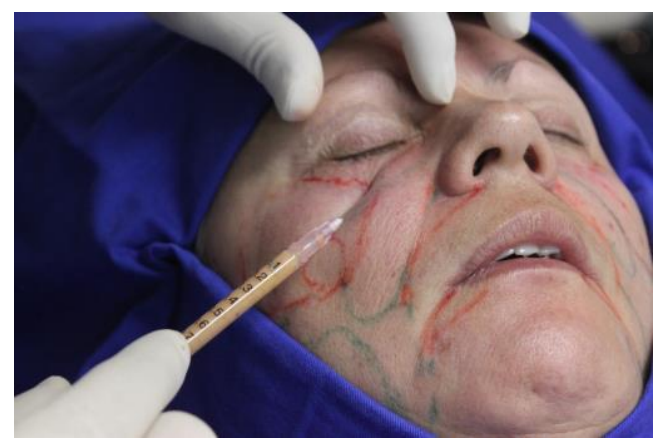

Figure 8. Tear trough filling beginning at the supraperiosteal plane

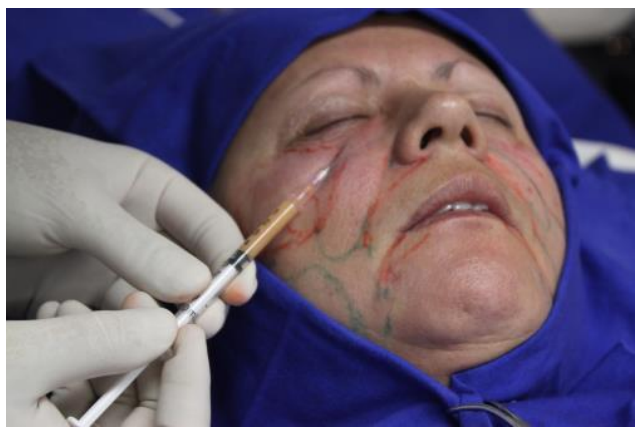

Figure 9. Uniform distribution is applied, always in small quantities, until the ideal volume and uniformity has been reached

The injection pressure must be minimal. In case of any resistance, it must not be forced, and the syringe and microcannula must be changed. This probably indicated a bigger fat fragment and could cause irregularities if 
injected under the skin. Care must be taken to avoid superficial irregularities, the main complication in difficult reversion. There is no need for hypercorrection as the fat graft frequently retains near $100 \%$. In the author's experience, definitive results are established at the second or third month after fat grafting. Any corrections can be done after this period.

In almost all cases, the lower lid blepharoplasty is performed, releasing only the skin, without touch the muscle, just like the pinch blepharoplasty technique ${ }^{[20]}$.

\section{Results}

\section{Anatomical study}

The following characteristics were found and are in agreement with Haddock et al. ${ }^{[16,17]}$, Hirmand ${ }^{[12]}$, Yang et al. ${ }^{[18]}$, and Yang et al. ${ }^{[19]}$.

(1) The tear trough and lid-cheek junction are not located at the bony orbital rim but are situated several millimeters caudally;

(2) The sulcus corresponds to the abrupt transition from the thin palpebral skin, without underlying subcutaneous fat, to the thicker orbital skin, with the presence of malar fat compartment (Figure 10);

(3) This transition is at the cranial limit of malar fat compartment;

(4) The tear trough is not caused by the volume of nasal alar and upper lip elevator muscle, which is more caudal and medial;

(5) The tear trough is located at the transition of the palpebral and orbital part of the orbicular muscle; medially, the bone attachment is the muscle origin with no ligaments found (Figure 11).

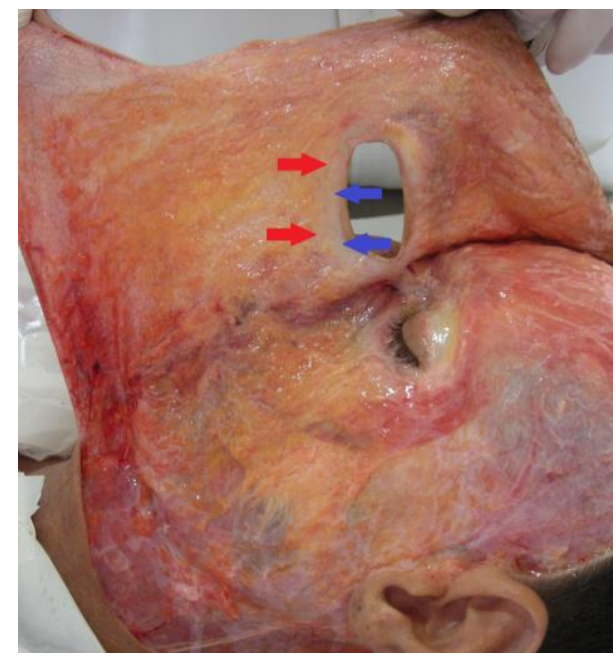

Figure 10. Palpebral skin without subcutaneous fat (blue arrows), and the abrupt transition to the malar skin and cranial limit for malar fat compartment (red arrows)

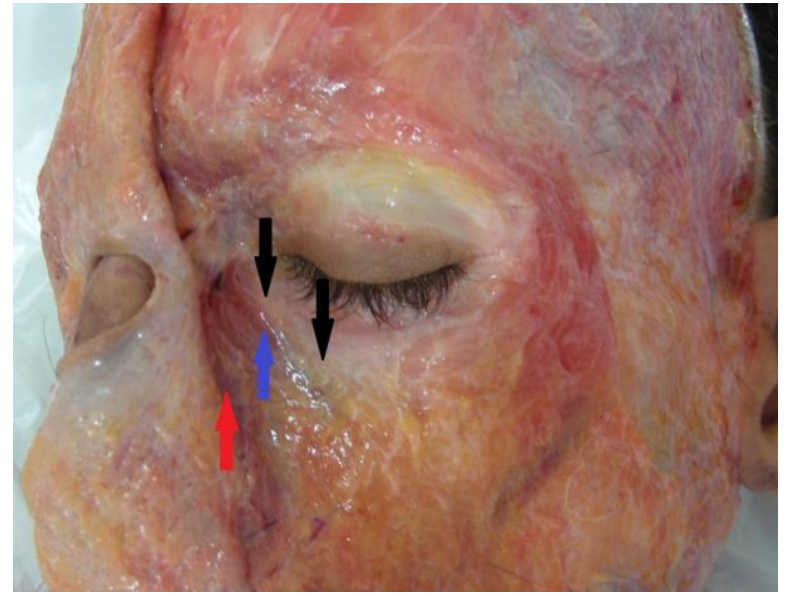

Figure 11. The tear trough (black arrow) is located caudally to the orbital rim on the OOM origin (blue arrow), superior and lateral to the nasal alar and upper lip elevator muscle (red arrow)

\section{Autologous fat grafting}

The last 200 consecutive procedures by a single surgeon were evaluated. Edema and bruises were spontaneously resolved in three or four weeks. Two patients with dark lower lid skin had worsening periorbital hyperpigmentation and needed dermatological treatment. One patient was a 23-year-old woman with acne after facial fat grafting. The other had previous festoons with prolonged malar edema and hypertrophic blepharoplasty scars. There were no other complications (Figures 12-16).
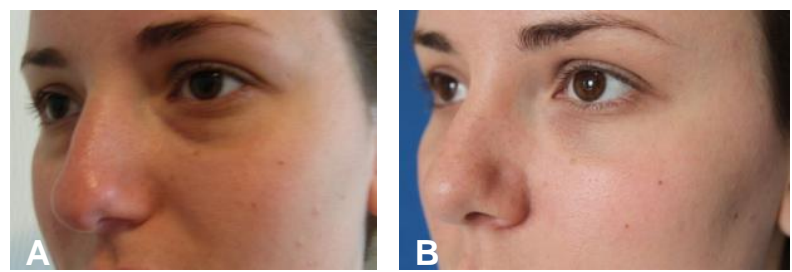

Figure 12. A young, 23-year-old patient with marked tear trough (A); Six months after periorbital and malar fat grafting (B)
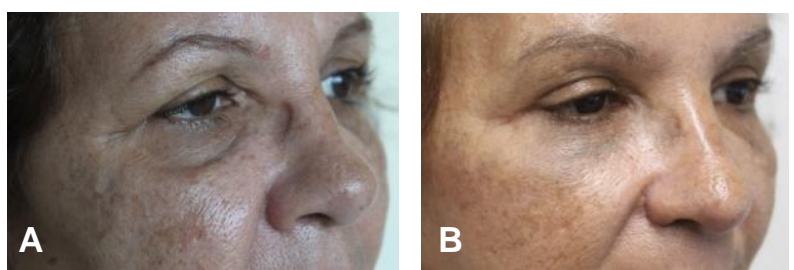

Figure 13. A 68-year-old patient with marked tear trough and lid-cheek junction (A); Seven months after blepharoplasty, periorbital, malar fat grafting, and full face lifting (B) 

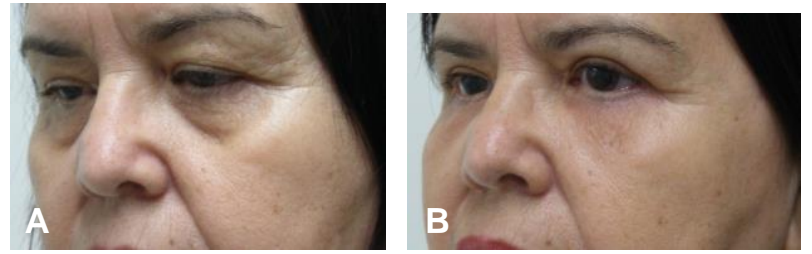

Figure 14. A 46-year-old patient with marked tear trough highlighting the palpebral fat pads (A); four months after blepharoplasty, periorbital, and malar fat grafting, without release of palpebral fat pads (B)
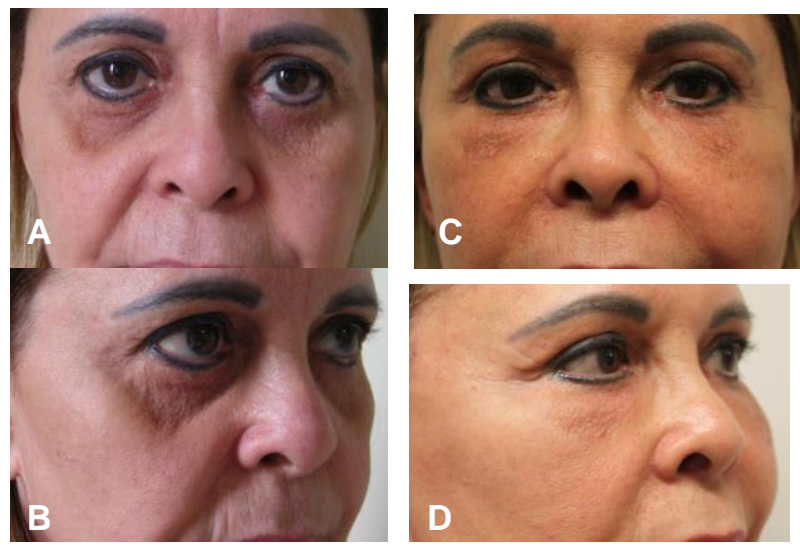

Figure 15. A 48-year-old patient with iatrogenic severe periorbital depression after three blepharoplasties (A and B); Six months after the $2^{\text {nd }}$ face lift and $4^{\text {th }}$ blepharoplasty, tarsal strip, periorbital, and malar fat grafting procedures were performed (C and D)
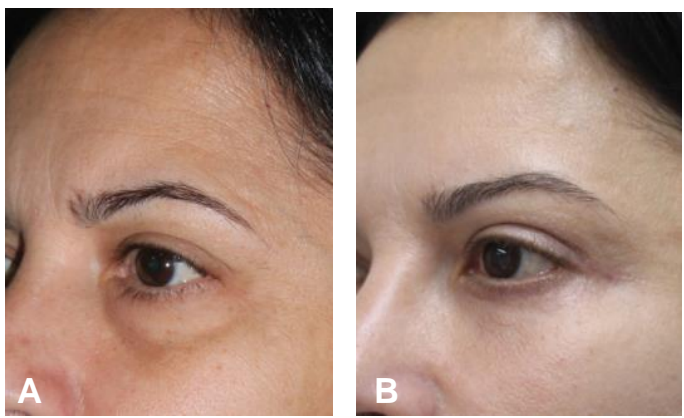

Figure 16. A 46-year-old patient presenting tear trough and lid-cheek junction (A); Four months after face lift, blepharoplasty, periorbital, and malar fat grafting (B)
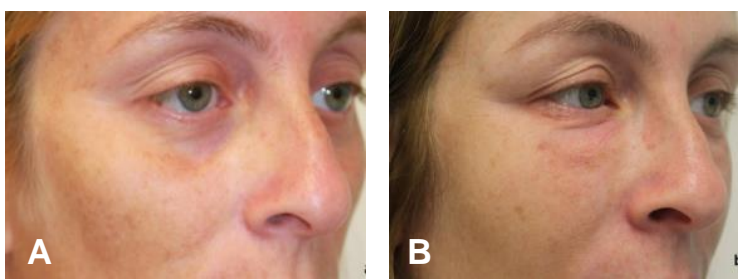

Figure 17. A 32-year-old thin patient with deep eyes (A); 15 months after periorbital fat grafting, $12 \mathrm{~kg}$ above her normal weight because of pregnancy, and grafted fat graft was also hypertrophic (B)

\section{Discussion}

The tear trough is frequently neglected in aesthetic facial treatments as the results are unpredictable, the procedure is difficult, the anatomy is complex and the tissue involved is delicate. Several explanations have been formulated for the cause of tear trough such as bony orbital rim; depression between the OOM, nasal alar and upper lip muscle; tissue descent by gravity, etc. ${ }^{[16]}$. The few anatomical studies in fresh cadaver dissections revealed that the main problem is subcutaneous fat ${ }^{[16-19]}$. The existence of a ligament was in doubt ${ }^{[21]}$. Our dissections proved that the attachment is of muscle origin ${ }^{[22]}$ and not a specific ligament.

Tear trough can be corrected using inferior palpebral medial fat pad transposition during blepharoplasty ${ }^{[23]}$ but it requires the presence of excess fat pad. This procedure is much more invasive and it only corrects the deep plane and not the superficial plane.

Alloplastic materials such as hyaluronic acid are a good option when patients want procedures which are more practical ${ }^{[24]}$. Fat grafting is the author's preference due to many reasons: the fat is abundant, the procedure is autologous, fat grafting is a cell transplant which has anti-inflammatory "anti-fibrotic" and "trophic" effects with a possibility of achieving better skin quality, and the results are natural ${ }^{[25-27]}$.

In literature, there is no difference in clinical results regarding the donor site, the use of anesthetic agents, vasoconstrictors, negative pressure, harvested fat process methods (centrifugation, decanting, washing, etc. ${ }^{[28,29]}$, supplementation with stromal vascular fraction ${ }^{[30]}$, or platelet rich plasma ${ }^{[31]}$.

In the author's experience, the most important influences are:

(1) Age: As a person gets older, the probability for success is smaller;

(2) Women usually have more predictable and better results;

(3) Tobacco has a negative influence; and

(4) Injection technique: Fine tunnels are well distributed in three dimensions, avoiding "bolus" injection.

In retrospect, the transplanted and retained volumes were not measured; the evaluation efficacy was made subjectively based on patient's and physician's satisfaction. The safety assessment was based on the complication rate. The grafted fat stabilized at the second or third month after the procedure and, in the author's experience, is in disagreement with literature that described stabilization at six months. In the patient evaluation, hyperpigmentation influenced more than the retained volume but 
the author was more demanding in the overall result, especially in uniformity for aesthetic unity. No other comparisons such as gender, age or body mass index were made, due to inadequate sampling. However, aesthetic results were excellent and quite uniform and the complication rate is extremely low, which is in agreement with literature ${ }^{[32,33]}$.

The periorbital sulcus is a natural anatomical depression of a personal characteristic, and it can be highlighted by aging. The pseudoptosis is one of the mechanisms of midface aging process and, according to Rohrich, is due to decreased volume of fat compartments especially at the medial deep malar fat compartment ${ }^{[6]}$. This lack of volume cannot be corrected with only face lifts or blepharoplasties. Extra volume filling is a simple and effective solution.

\section{Conclusion}

The main mechanism of midface aging process is the deflation phenomenon. It must be specifically treated by extra volume filling, and not only with surgeries. Despite not having measured the retained volume, the treatment of tear trough by autologous fat grafting seems effective and safe, and may be more advantageous than alloplastic materials. Anatomically, there is no specific plane for volume injection. For uniformity of the palpebromalar region, the fat graft must be evenly distributed in deep and superficial planes.

\section{Conflict of interest}

The author declared no potential conflict of interest with respect to the research, authorship, and/or publication of this article.

\section{References}

1. Goldberg RA. The three periorbital hollows: A paradigm for periorbital rejuvenation. Plast Reconstr Surg 2005; 116(6): 1796-1804. doi: 10.1097/01.prs.000018562 3.367 95.38.

2. Pessa JE, Desvigne LD, Lambros VS, Nimerick J, Sugunan $\mathrm{B}$, et al. Changes in ocular globe-to-orbital rim position with age: Implications for aesthetic blepharoplasty of the lower eyelids. Aesth Plast Surg 1999; 23(5): 337342. doi: 10.1007/s002669900295.

3. Donofrio LM. Fat distribution: A morphologic study of the aging face. Dermatol Surg 2000; 26(12): 1107-1112. doi: 10.1046/j.1524-4725.2000.00270.x.

4. Lambros V. Observations on periorbital and midface aging. Plast Reconstr Surg 2007; 120(5): 1367-1376. doi: 10.1097/01.prs.0000279348.09156.c3.
5. Rohrich RJ, Pessa JE. The fat compartments of the face: Anatomy and clinical implications for cosmetic surgery. Plast Reconstr Surg 2007; 119(7): 2219-2227. doi: 10.1097/01.prs.0000265403.66886.54.

6. Rohrich RJ, Pessa JE, Ristow B. The youthful cheek and the deep medial fat compartment. Plast Reconstr Surg 2008; 121(6): 2107-2112. doi: 10.1097/PRS.0b013e318 $17123 \mathrm{c} 6$.

7. Pessa JE. An algorithm of facial aging: Verification of Lambros's theory by three-dimensional stereolithography, with reference to the pathogenesis of midfacial aging, scleral show, and the lateral suborbital trough deformity. Plast Reconstr Surg 2000; 106(2): 479-488. doi: 10.1097/00006534-200008000-00040.

8. Pessa JE, Chen Y. Curve analysis of the aging orbital aperture. Plast Reconstr Surg 2002: 109(2): 751-760.

9. Shaw RB Jr, Kahn DM. Aging of the midface bony elements: A three-dimensional computed tomographic study. Plast Reconstr Surg 2007; 119(2): 675-681. doi: 10.1097/01.prs.0000246596.79795.a8.

10. Mendelson BC, Hartley W, Scott M, McNab A, Granzow JW. Age-related changes of the orbit and midcheek and the implications for facial rejuvenation. Aesth Plast Surg 2007; 31(5): 419-423. doi: 10.1007/s00266-006-0120-x.

11. Yaremchuk MJ, Kahn DM. Periorbital skeletal augmentation to improve blepharoplasty and midfacial results. Plast Reconstr Surg 2009; 124(6): 2151-2160. doi: 10.1097/PRS.0b013e3181bcf5bc.

12. Hirmand H. Anatomy and nonsurgical correction of the tear trough deformity. Plast Reconstr Surg 2010; 125(2): 699-708. doi: 10.1097/PRS.0b013e3181c82f90.

13. Yaremchuk MJ. Infraorbital rim augmentation. Plast Reconstr Surg 2001; 107(6): 1585-1592. doi: 10.1097/0000 6534-200105000-00047.

14. Trepsat F. Periorbital rejuvenation combining fat grafting and blepharoplasties. Aesth Plast Surg 2003; 27(4): 243-253. doi: 10.1007/s00266-003-2126-y.

15. Trepsat F. Lipostructure du tiers moyen du visage (French) [Midface reshaping with micro-fat grafting]. Ann Chir Plast Esth 2009; 54(5): 435-443. doi: 10.1016/j.anplas.20 09.03.008.

16. Haddock NT, Saadeh PB, Boutros S, Thorne CH. The tear trough and lid/cheek junction: Anatomy and implications for surgical correction. Plast Reconstr Surg 2009; 123(4): 1332-1340. doi: 10.1097/PRS.0b013e31819f2b36.

17. Pessa JE. Discussion: The tear trough and lid/cheek junction: Anatomy and implication for surgical correction. Plast Reconstr Surg 2009; 123(4): 1341-1342. doi: 10.1097/PRS.0b013e31819f25ec.

18. Yang N, Qiu W, Wang Z, Su X, Jia H, et al. (Chinese) [Anatomical studying of the tear trough area]. Zhonghua 
Zheng Xing Wai Ke Za Zhi 2014; 30(1): 50-53.

19. Yang C, Zhang PP, Li JH, Xing X. (Chinese) [Anatomic study on the mechanism of tear trough deformity and palpbromalar groove deformity]. Zhonghua Zheng Xing Wai Ke Za Zhi 2010; 26(2): 139-142.

20. Rosenfield LK. The pinch blepharoplasty revisited. Plast Reconstr Surg 2005; 115(5): 1404-1412. doi: 10.1097/0 1.PRS.0000157020.67216.31.

21. Wong $\mathrm{CH}$, Hsieh $\mathrm{MKH}$, Mendelson B. The tear trough ligament: Anatomical basis for the tear trough deformity. Plast Reconstr Surg 2012; 129(6): 1392-1402. doi: 10.1097/PRS.0b013e31824ecd77.

22. Pessa JE. Discussion: The tear trough ligament: Anatomical basis for the tear trough deformity. Plast Reconstr Surg 2012; 129(6): 1403-1404. doi: 10.1097/PRS.0b013e $3182507 \mathrm{a} 08$.

23. Youn S, Shin JI, Kim JT, Kim YH. Transconjunctival subperiosteal fat reposition for tear trough deformity: Pedicled fat redraping versus septal reset. Ann Plast Surg 2014; 73(5): 479-484. doi: 10.1097/SAP.0b013e318 $27 \mathrm{f} 5455$.

24. Hill RH 3rd, Czyz CN, Kandapalli S, Zhang-Nunes SX, Cahill KV, et al. Evolving minimally invasive techniques for tear trough enhancement. Ophthal Plast Reconstr Surg 2015; 31(4): 306-309. doi: 10.1097/IOP.0000000000000 325.

25. Chang YC, Rovaris DA. Lipoenxertia autóloga periorbitária no rejuvenescimento facial: Análise retrospectiva da eficácia e segurança em 31 casos (Portuguese) [Autologous periorbital fat grafting in facial rejuvenation: A retrospective analysis of efficacy and safety in 31 cases]. Rev Bras Cir Plást 2012; 27(3): 405-410. doi: 10.1590/S 1983-51752012000300013.

26. Majollal A, Lequeux C, Shipkov C, Breton P, Foyatier JL, et al. Improvement of skin quality after fat grafting: Clinical observation and an animal study. Plast Reconstr Surg 2009; 124(3): 765-774. doi: 10.1097/PRS.0b013e31 $81 \mathrm{~b} 17 \mathrm{~b} 8 \mathrm{f}$
27. Covarrubias P, Cárdenas-Camarena L, Guerrerosanto J, Valenzuela L, Espejo I, et al. Evaluation of the histologic changes in the fat-grafted facial skin: Clinical trial. Aesth Plast Surg 2013; 37(4): 778-783. doi: 10.1007/s00266-01 3-0126-0.

28. Gir P, Brown AS, Oni G, Kashefi N, Mojallal A, et al. Fat grafting: Evidence-based review on autologous fat harvesting, processing, reinjection, and storage. Plast Reconstr Surg 2012; 130(1): 249-258. doi: 10.1097/PRS.0b013 e318254b4d3.

29. Rochrich RJ, Sorokin ES, Brown SA. In search of improved fat transfer viability: A quantitative analysis of the role of centrifugation and harvest site. Plast Reconstr Surg 2004; 113(1): 391-395. doi: 10.1097/01.PRS.00000 97293.56504.00.

30. Mailey B, Saba S, Baker J, Tokin C, Hickey S, et al. A comparison of cell-enriched fat transfer to conventional fat grafting after aesthetic procedures using a patient satisfaction survey. Ann Plast Surg 2013; 70(4): 410-415. doi: 10.1097/SAP.0b013e31827e5353.

31. Sagarello M, Visconti G, Rusciani A. Breast fat grafting with platelet-rich plasma: A comparative clinical study and current state of the art. Plast Reconstr Surg 2011; 127(6): 2176-2185. doi: 10.1097/PRS.0b013e3182139f e7.

32. Kim SM, Kim YS, Hong JW, Roh TS, Rah DK. An analysis of the experiences of 62 patients with moderate complications after full-face fat injection for augmentation. Plast Reconstr Surg 2012; 129(6): 1359-1368. doi: 10.1097/PRS.0b013e31824ecbb0.

33. Grahovac TL, Rubin JP. Discussion: An analysis of the experiences of 62 patients with moderate complications after full-face fat injection for augmentation. Plast Reconstr Surg 2012; 129(6): 1369-1370. doi: 10.1097/PRS. 0b013e31824f4ffc. 